\title{
AGN Selection by Size of Dominant Emission
}

\author{
Pedro Augusto \\ Universidade da Madeira, Centro de Ciências Matemáticas, Caminho \\ da Penteada, 9000-390 Funchal, Portugal

\section{J. Ignacio Gonzalez-Serrano} \\ Instituto de Física de Cantabria (CSIC-Universidad de Cantabria), \\ Facultad de Ciencias, 39005 Santander, Spain
}

\begin{abstract}
The potential of AGN surveys extends to structure-related objectives: e.g. to study the size and properties of the NLR in AGN. From a complete sample of 1665 radio sources, we selected fifty-five with features on $\sim 0.2-2 \mathrm{kpc}$ scales (core/jet flux ratio $<7: 1$ ) for further study. Here, we summarize the radio interferometric selection technique used and speculate on the prospects for optical surveys (using a size criterion).
\end{abstract}

\section{Introduction}

Typically, brightness related criteria (only) are used to select sub-samples from AGN surveys. Why is a physical size selection criterion (almost) never adopted? Such selection is particularly useful when used in statistical/systematic studies of some type of AGN (or regions within) with a given size range; also, to determine the type of AGN associated with some physical phenomenon (dependent on size). A careful size selection has relevant scientific output (see Section 3).

The standard model of AGN, so far consistent with observations, besides two powerful opposing radio emitting jets, contains a (likely) spherically symmetric NLR, even though we normally only see the excited NLR cones (e.g. Mrk 3 in Cappetti et al. 1996). The radius of the NLR is in the range 0.01-1 $\mathrm{kpc}$, although it has been well studied only in nearby Seyfert galaxies $(z \lesssim 0.2)$, namely by using the HST. Correlations of the NLR with the radio jets have been identified (e.g. Augusto et al. 2001); simulations of jets going through physical interfaces such as the NLR/rest of the ISM show that it is possible to associate certain radio features with the physical size of the NLR. However, since only nearby Seyferts had NLR studies done, the sample of Section 3 is an example of how to approach the answer to questions such as: How is the NLR in the rest of the Universe? is it omnipresent in all AGN types? is its size always the same? are its physics the same? is there any evolution, perhaps correlated with the general AGN evolution? How does the standard model change when we know more about the NLR evolution? 


\section{The $\sim$ Kpc-Scale Phenomena in AGN}

\subsection{CSO/MSOs}

Compact (medium) symmetric objects (CSO/MSOs) show flat or inverted spectra $\left(\alpha<0.5 ; \mathrm{S}_{\nu} \propto \nu^{-\alpha}\right)$ and have symmetric components (lobes) on each side of a putative core. CSOs are smaller than $1 \mathrm{kpc}$, while MSOs have sizes of 1-20 kpc. There are many cases of ultra-small pc-scale CSOs (VLBI scale; e.g. Taylor et al. 1996) and a few candidates for large sub-kpc CSOs (Augusto et al. 1999). A few CSOs had kinematics studied (e.g. Owsianik, Conway, \& Polatidis 1998) showing them to have typical ages of $\sim 10^{3} \mathrm{yrs}$. If they are the early stages of FRII radio galaxies, Augusto, Wilkinson \& Browne (1998) have shown them to evolve self-similarly, i.e., their lobes expanding as they grow and most ${ }^{1}$ becoming Compact Steep Spectrum Sources (CSSs) when reaching sub-kpc sizes.

\subsection{NLR Probes}

CSO/MSOs are excellent probes of the NLR/ISM interface, since they likely lie in the plane of the sky (unbeamed, symmetrical structure). Finding their redshifts and studying them better could mean understanding the NLR over the billions of years of evolution of AGN.

As regards other types of objects, e.g. core-plus-one-sided-jets (CJs), orientation issues are of more concern, although these can be solved with enough data (Fig. 1). Their potential as NLR probes, however, is improved when they present not only features (knots and strong blobs) on the right scales for a NLR/ISM interface (e.g. Fig. 1 in Augusto et al. 2001) but also heavily bent jets $\left(>90^{\circ}\right)$ : hint of strong shocks which should be confirmed from radio polarization and optical spectroscopy observations.

By probing the NLR with radio jets at different redshifts we can learn about: i) the size of the NLR; ii) the actual sub-structure of the NLR (cloud formations, etc.) - from radio shocks; iii) the (mean) density of the gas clouds; iv) bolometric emission characteristics; v) importance of magnetic fields - from radio polarization maps; vi) all of $\mathrm{i}$ )- $\mathrm{v}$ ) in redshift space, tracing the evolution of the NLR in several parameters.

\section{Sampling by Size: an Example on $\sim 0.2-2 \mathrm{Kpc}$ Scale}

\subsection{The Sample Selection}

Starting from a parent sample of all radio sources in the northern sky with $\mathrm{S}_{8.4 \mathrm{GHz}}>100 \mathrm{mJy}, \alpha_{1.4}^{4.85}<0.5$ and $\left|b^{I I}\right|>10^{\circ}$ (totaling 1665 objects), Augusto et al. (1998) have selected a sub-sample of 55 radio sources with structure on 90 300 milliarcseconds (mas) scale, which translates into projected linear structures (strong multiple radio features with $<7: 1$ flux density ratio) of $\sim 0.2-2 \mathrm{kpc}$ at $z>0.2$.

\footnotetext{
${ }^{1}$ As for MSOs, they could be the descendents of the few CSOs that reach maturity without getting through the CSS stage.
} 


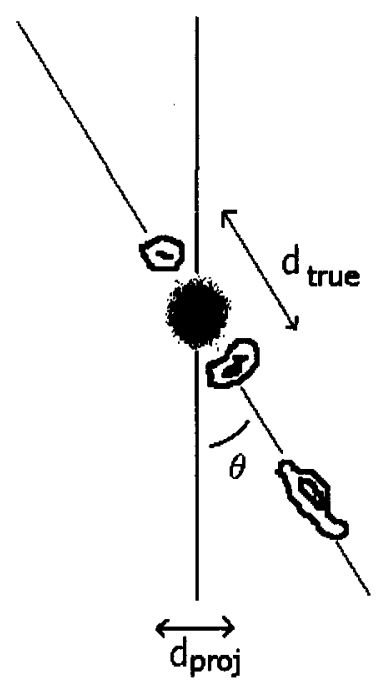

Figure 1. Knowing the orientation of a radio source is essencial for translating projected sizes of the NLR into actual sizes (dproj $\leq$ dtrue). For core-jets we can (e.g.): a) determine the Lorentz factor, if a counterjet is detected, and beaming exists (likely); b) use limb brightening of the jets/blobs; c) use polarization effects.

As described in Augusto et al. (1998), the size selection was not made by looking at the radio maps of the 1665 radio sources but rather by looking at their radio visibilities. Any radio interferometer can actually 'see' below the formal beam (resolution). At $8.4 \mathrm{GHz}$, the VLA-A (which was used in this case) has a resolution of $\sim 200$ mas and yet Augusto (1996) has shown that a compact 50 mas equal double can actually be detected via visibility, although it is impossible to see more than an unresolved source in the map. However, this detection depends on the observing conditions (namely elevation of source Augusto et al. 1998) and it may not work all the time. This is why, with $100 \%$ confidence, only 90 mas equal doubles are detectable from VLA-A visibilities (Augusto et al. 1998; Fig. 2).

The techique used for detecting 50 mas doubles (or larger) from the visibilities was as shown in Fig. 3. In the case of a double radio compact source with components $S_{1}$ and $S_{2}$ where $S_{1} \leq 7 S_{2}$ we have (interferometry theory) $S_{\text {peak }}=S_{1}+S_{2} \leq 8 S_{2}$ and $S_{\text {min }}=S_{1}-S_{2} \geq 6 S_{2}$. Hence:

$$
\frac{S_{\text {peak }}-S_{\text {min }}}{S_{\text {peak }}}=1-\frac{S_{\text {peak }}}{S_{\text {min }}} \geq 1-\frac{6}{8}=0.25
$$

So, all we did was to look for sources that showed a greater than $25 \%$ decrease in correlated flux from the shorter to the longer baselines.

\subsection{Observational Status}

An extensive follow up has been conducted in the optical (imaging - GonzalezSerrano et al., in prep.) and radio (high resolution dual frequency maps Augusto et al., in prep.) for most of the 55 objects. We also have $22 \mathrm{GHz}$ data 


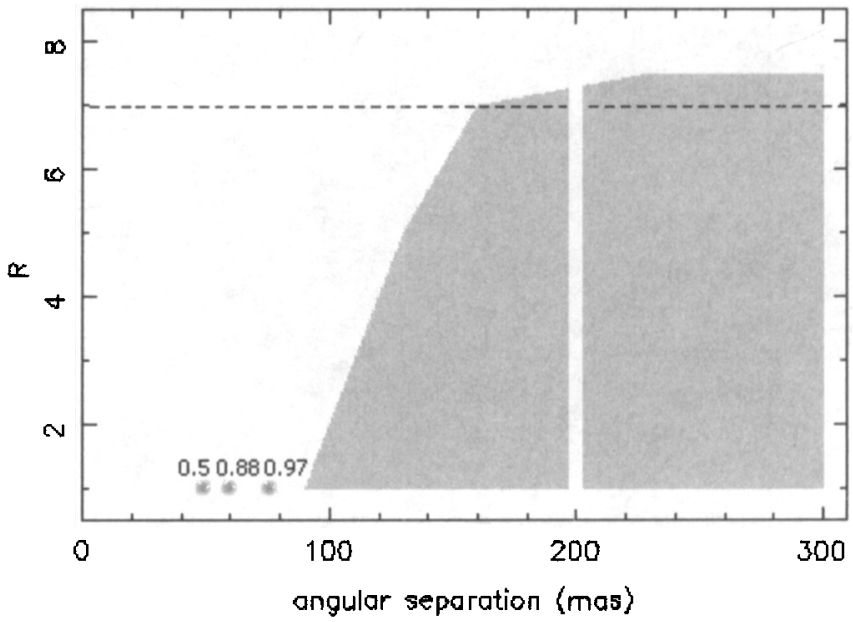

Figure 2. The size selection of compact doubles with flux density ratio $R$ as function of separation. The grey area means $100 \%$ reliability, while the fraccional reliabilities (labels) refer to equal doubles. The VLA-A $8.4 \mathrm{GHz}$ formal beam is marked (white line at 200 mas). Note that larger-than-the-beam compact doubles with $\lesssim 7.5: 1$ flux density ratio are also detected (adapted from Augusto et al. 1998).

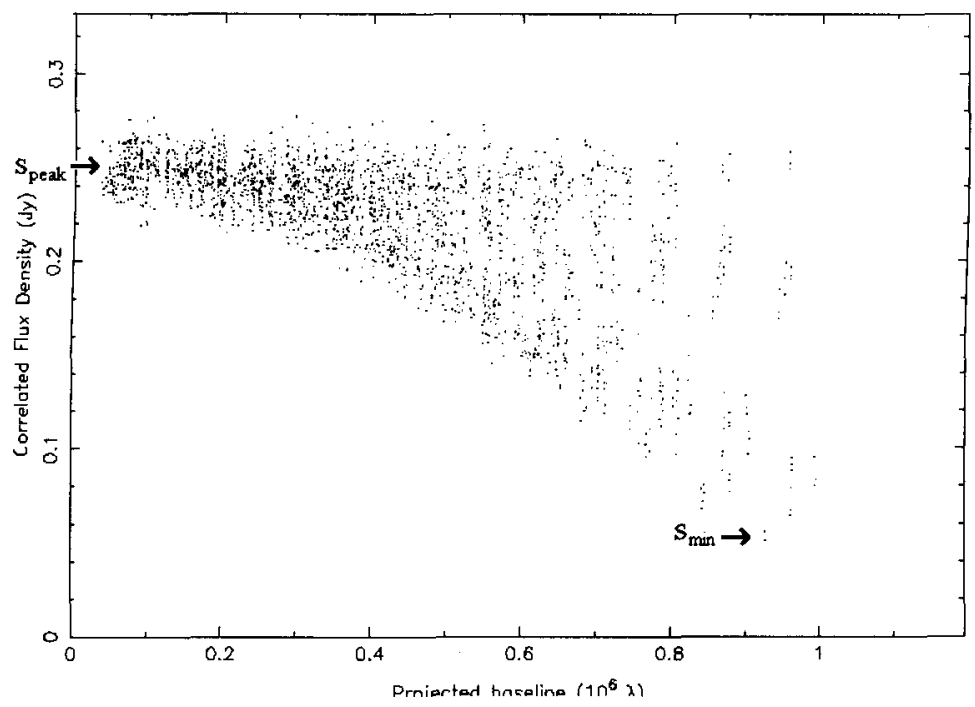

Figure 3. The technique for selecting radio sources by size on 90300 mas (with $100 \%$ confidence - see Fig. 2). This was looking for sources with a $\geq 25 \%$ decrease in correlated flux from the shorter to the longer baselines: $\left(S_{\text {peak }}-S_{\text {min }}\right) / S_{\text {peak }} \geq 0.25$. Note that $S_{\text {min }}$ may not necessarily correspond to the longest baselines (adapted from Augusto 1996). 
(VLA, MERLIN) on some objects. Reduction of this extensive amount of data is underway. Preliminary optical results indicate that, of the 55 objects, 3 are fainter than 23 mag. Their morphology is not clear, since most appear too small for ground-based telescope observations.

A spectroscopic programme, vital for redshift determination and shock confirmations, has yet to be started. Nevertheless, we can make a preliminary analysis of the (biased) literature information existing for 20 of the 55 sources. At the moment we get $\langle z\rangle=0.66 \pm 0.64$ suggesting a medium-to-high redshift sample of objects with large spread. In fact, the redshift range is $0.015 \leq z \leq 2.249$ with a Sy2 galaxy and a QSO at the closer and farther extremes, respectively. The most distant confirmed radio galaxy in the sample is at $z=1.152$. It is then probable that the overall average redshift of the sample of 55 sources will be larger than 1. More important is the fact that a large spread exists and thus we might be able to probe the evolution of the NLR in AGN (and testing AGN models in general) in redshift space, as intended.

\section{Size Selection}

\subsection{Its Potential}

We have just shown relevant scientific output from a size selection. Although this paper (like the Colloquium) is on Extragalactic Astronomy (AGN), we should stress that any size selection from surveys can have the specific aim of Galactic objects and phenomena. In Section 3 we have virtually excluded 'contamination' by Galactic objects $\left(\left|b^{I I}\right|>10^{\circ}\right.$ was one of the criteria imposed to get the 1665source sample from the original surveys). However, we might be interested, for example, in finding very young Supernova Remnants in our galaxy (flat spectrum and small). In Table 1 we show the range of astronomy exploration that can be made, even unintentionally, with a size selection.

Table 1. The Potential of Size Selection for Different Areas of Astronomy.

\begin{tabular}{cll}
\hline & \multicolumn{2}{c}{$0^{\prime \prime} 5-0^{\prime} 3$ angular size selection } \\
\hline Linear projected size & \multicolumn{1}{c}{ Distance (example) } & \multicolumn{1}{c}{ Type of study (example) } \\
\hline$\sim 0.2-2 \mathrm{kpc}^{a}$ & $z \sim 0.5$ (AGN) & NLR probes \\
$\sim 4-24 \mathrm{pc}$ & $\sim 20 \mathrm{Mpc}$ (Virgo Cluster) & Supernova Remnants (SNR) \\
$\sim 0.2-1.2 \mathrm{pc}$ & $\sim 1 \mathrm{Mpc}$ (Andromeda galaxy) & very young SNR \\
$\sim 40-240 \mathrm{AU}$ & $\sim 1 \mathrm{kpc}$ (Galaxy) & Stellar wind, planets, IPM/ISM \\
\hline \hline
\end{tabular}

${ }^{a}$ Depends on cosmological model. All currently accepted models are included in this order-ofmagnitude range.

\subsection{At Different Wavelengths}

Radio There are many advantages in selecting radio sources by size using 'raw' radio interferometric data (calibrated only), before any CLEANing is done (the 

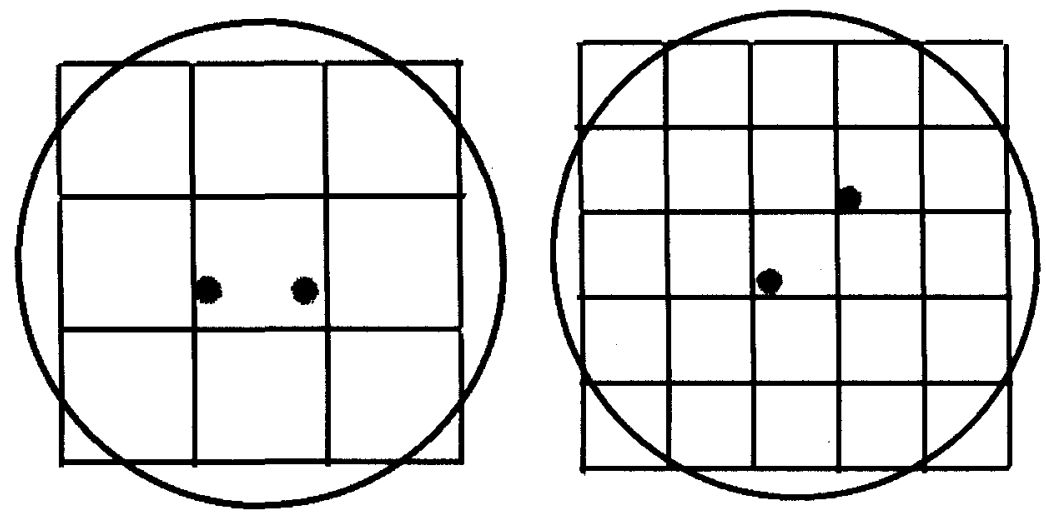

Figure 4. (left) Using pixels with a size of 60 mas, a 50 mas compact double may fit inside one pixel (the VLA-A $8.4 \mathrm{GHz}$ formal beam is marked with a circle 200 mas in diameter). (right) This time we use 35 mas pixels making sure that a 50 mas compact double will never have both components in the same pixel.

most popular algorithm for producing radio maps). For example, although the VLA-A $8.4 \mathrm{GHz}$ can 'see' down to a 50 mas size (separation) — Section 3.1, this structure is only found on the visibility data, either visually (e.g. Fig. 3) or by automatically applying models.

An example of how information still can be preserved has to do with the so-called superresolution: typically, precisely because a sensitive radio interferometer like the VLA can 'see' smaller than the formal beam, we can get a feeling of smaller-than-the-beam radio structure by convolving the final CLEANed data with a beam smaller than the natural beam (e.g. for VLA-A $8.4 \mathrm{GHz}$ maybe a 70 mas beam). Although risking misinterpretation of radio features if taken too seriously, superresolution allows us to know how really unresolved a given radio source (component) is, without having to look at the radio visibility. CLEANing usually starts off with deciding on the pixel size for the algorithm. This, typically, is about one-third of the formal beam, i.e., $\sim 60$ mas for the VLA-A 8.4 $\mathrm{GHz}$. However, using this size of pixels will elude 'detection' with superresolution of, e.g., any $\sim 50$ mas compact equal doubles, even though they are clearly seen in visibility (Augusto et al. 1998) - Fig. 4. On the other hand, using smaller pixels ( $\leq 35$ mas) may increase the computational time for reducing the data but will allow detection of $\sim 50$ mas compact doubles using superresolution (Fig. 4), since each component now always ocupies different pixels. To prevent aliasing by inefficient sampling, the pixels cannot be too small, however.

Optical (philosophy) It is obvious that interferometry should work pretty much the same as radio interferometry and so, detection of structure smaller than the formal resolution will also be possible in sensitive instruments. As regards 
single-telescope use (with CCD), we must split into; 1) ground-based and 2) space-based:

1. although the pixels are, e.g., $0^{\prime \prime} 1$ on a side, the seeing limits the resolution to, say, $0^{\prime}$ ' 6 ; worse than that, there is a complex rapid-time-varying 'beam' resulting from the convolution of the PSF of a point source and the seeing effect. With the use of 'smart' adaptive optics, we may be able not only to compensate the seeing (as it is currently done successfully) but also to go below the formal resolution of the instrument (using smaller CCD pixels and a way to get extra-information on the source from the complex 'beam'); at the moment we certainly can use wide field, large pixel (e.g. 0.6) CCDs to go around the seeing problem and separate structure (compact doubles: binary stars, for example). For extended sources, we may play around with isophotes, modeling them and 'guessing' structure smaller than 0 "' 6 , even without the use of adaptive optics;

2. HST is already using the dithering technique which allows a slight improvement in resolution; when we are working with single pixels, however, the issue of cosmic-ray contamination becomes relevant and we must exercise care for detecting $\sim 0^{\prime \prime} 05-0{ }^{\prime} 1$ structure, distinguishing it from cosmic rays - either using several frames (as it is done currently in most observations) or by simulating the likelihood of neighboring events.

Acknowledgments. We acknowledge the grant from the Fundação para a Ciência e a Tecnologia (ESO Programme): PESO/P/PRO/15133/1999. The European Commission, under contract ERBFMGECT 950012, gave us partial support for this research.

\section{References}

Augusto, P. 1996, Ph.D. thesis, Univ. Manchester, UK

Augusto, P., Wilkinson, P. N., \& Browne, I. W. A. 1998, MNRAS, 299, 1159

Augusto, P., Gonzalez-Serrano, J. I., Edge, A. C., Gizani, N. A. B., Wilkinson, P. N., \& Perez-Fournon, I. 1999, New Astron. Rev., 43, 663

Augusto, P., Gonzalez-Serrano, J. I., Gizani, N. A. B., Perez-Fournon, I., \& Edge, A. C. 2001, Astron. \& Astroph. Trans., in press

Cappetti, A., Axon, D. J., Macchetto, F., Sparks, W. B., \& Boksenberg, A. 1996, ApJ, 469, 554

Owsianik, I., Conway, J. E., \& Polatidis, A.G. 1998, A\&A, 336, L37

Taylor, G. B., Vermeulen, R. C., Readhead, A. C. S., Pearson, T. J., Henstock, D. R., \& Wilkinson, P. N. 1996, in: Snellen, I., Schilizzi, R. T., Rottgering, H. J. A., \& Bremer, M. N. (eds), $2^{\text {nd }}$ Workshop on Gigahertz Peaked Spectrum and Compact Steep Spectrum Radio Sources, Univ. Leiden 